\title{
INTERPRETATION OF SERUM REACTIONS IN LATE SYPHILIS*
}

\author{
BY \\ C. P. HEYWOOD \\ Bradford
}

Two problems confront the venereologist in his interpretation of serum reactions in late syphilis. A satisfactory solution to the first, that of diagnosis, removes many of the difficulties of the second, that of the significance to be attached to the behaviour of the serum reactions during and after treatment.

\section{Diagnosis}

The problem here can be simplified till it reaches the commonplace. Theoretically, our interpretation of the results of serological tests in syphilis is handicapped by doubts as to the real nature of the tests we employ, of which more will be said later. But experience has proved that, whatever their fundamental nature, present-day serological tests for syphilis (S.T.S.) are, in practice, both highly sensitive and highly specific; and with appropriate safeguards to exclude temporarily false positive reactions, a positive reaction means that the patient has been infected with syphilis at some time in his life.

It is not a guide as to whether the patient is sick at the time, nor even as to whether he will ever become sick (Moore, 1946).

A quantitative serological test may provide a very rough indication of the age of the infection, a positive reaction to a high titre indicating a more recent infection, and a positive reaction to only a low titre indicating an infection of longer duration, because of the general tendency towards spontaneous reversal with the passage of time.

The positive serological test is nevertheless not a complete diagnosis upon which to base the further management of the case, but a stimulus to fuller investigation. Preferably this examination should be carried out by an experienced syphilologist, who can supplement the general examination already

* Address delivered to the Medical Society for The Study of Venereal Diseases, October 26, 1951. made by his special knowledge of the possibilities of the disease ; it should include (as summarized by Moore, 1946), a detailed medical history with uninhibited reference to the possibilities of venereal infection, a thorough physical examination, with special emphasis upon the cardiovascular and central nervous systems, and laboratory tests and $x$-ray examinations as necessary, but including as a minimum an examination of the cerebrospinal fluid (cell count, protein, Lange and Wassermann reactions), and a chest $x$ ray. In the course of this investigation the symptoms and signs, if any, which provoked it will be assessed ; these may be unrelated to the syphilitic infection, and the distinction may have to be made on clinical rather than serological grounds. One of the crosses we have to bear is the tendency of many patients and their physicians to ascribe every ailment to syphilis once a positive serological reaction has been discovered.

In the older literature there is frequent reference to the occurrence of untreated late syphilis in association with negative serological tests, but with the more highly sensitive tests available today the diagnostic problem so created should rarely arise ; when it does, it is, as always, resolved on clinical and therapeutic grounds. Before leaving this subject some mention must be made of another concept that has occupied much space of recent years, that of the so-called " biological false positive reaction" to serological tests for syphilis. This supposes that in the blood of a few entirely normal people (as distinct from patients suffering from a number of acute and chronic diseases known temporarily to produce a false positive S.T.S.), there may be sufficient reagin to produce a positive reaction. This concept belongs to the realm of armchair metaphysics and has no practical importance in the absence of any readily available method of distinguishing between the patient with the hypothetical biological false positive reaction and the 
patient with latent syphilis, other than the former's supposedly blameless life, a distinction shared with congenital syphilitics. However, it may serve a useful purpose in explaining the bishop's blood-test, provided that the physician does not neglect to investigate and treat him as if he were suffering from a latent syphilitic infection.

Having arrived at an accurate assessment of the patient's status vis-à-vis his syphilitic infection, the venereologist is in a position to plan his management of the case. This is outside the scope of the present discussion, but the golden rule in formulating this plan should be to treat the patient first, the disease second, and the serological reactions not at all (Scholtz, 1934).

The amount of treatment necessary is not measured by the blood-test. In most people who have had syphilis for more than two years before treatment is begun, the blood-test may never become negative, no matter how much or what kind of treatment is given. In elderly persons-over the age of 65-it may be undesirable to give any treatment for syphilis at all, even if the S.T.S. is positive, provided that investigation has revealed no other evidence of active infection (Moore, 1946).

\section{Behaviour of the Serum Reactions during and after Treatment}

It is interesting to follow the fate of untreated syphilitics as a preliminary. According to Bruusgaard (1929), these cases fall into four groups of approximately equal numerical importance :

(1) spontaneous cure, i.e. complete freedom from all detectable clinical, serological, and even post-mortem evidence of syphilis ;

(2) prolonged latency, i.e. absence of all clinical manifestations of syphilitic disease but persistence of a positive serological test for syphilis ;

(3) development of benign tertiary lesions, in the form of gummatous lesions of skin, mucous membranes, bones, and eyes ;

(4) very gradual development of degenerative and potentially killing or crippling late manifestations of cardiovascular or neuro-syphilis.

Rosahn (1946), in an investigation of the end results of untreated syphilis from post-mortem material, and Pesare, Bauer, and Gleeson (1950), in their periodical surveys of the results of untreated syphilis in a male Negro community in Alabama, report essentially similar results.

From Bruusgaard's figures it appears that approximately 70 per cent. of untreated syphilitics remain sero-positive, but by no means all of these develop late manifestations of syphilis. It is to be expected that the results in adequately treated late syphilis will be better than in untreated cases. The general tendency of the serum reactions during and after treatment is towards negativity, or at least towards a reduction in titre, though numerous writers acknowledge the persistence of positive serum reactions in supposedly adequately treated patients. What constitutes adequate treatment must be arbitrarily decided by each venereologist upon the results of his own and his colleagues' experience, based on the life-long observation of large numbers of patients. Only those patients should be deemed sero-resistant who retain a positive serum reaction after adequate treatment and in the absence of all other evidence of the disease. The significance to be attached to these persistently positive reactions provides the crux of the problem.

If they arise from a focus of spirochaetes sealed off from the influence of treatment, perhaps in an insignificant situation, the reagin being the product of the breakdown of the organisms, the interpretation of the sero-resistant patient's situation must be somewhat different from that which would prevail if the irreversible positive reaction could be regarded as a permanent metabolic change in tissue previously infected with syphilis but no longer harbouring the organisms. In the former, the positive test means active defence, organisms in process of destruction (Stokes, 1944). On this hypothesis, it is not quite clear how reagin from such a focus can reach the circulation, while the focus yet remains inaccessible to an effective remedy in the circulation (Hämel, 1934). Nor is it clear why such a focus is of any graver import than the focus of tuberculosis said to exist in all adults over the age of 25 , as a result of which they give positive reactions to skin tests for tuberculosis. If, on the other hand, seroresistance is due to a permanent metabolic change, or to the engendering, by longstanding infection, of immunity sufficiently strong to maintain clinical latency and to prevent progression or relapse (Kolmer, 1938), the positive serological reaction becomes of no more importance than a scar, or a positive Widal reaction after typhoid fever. Until the source of the reagin is definitely known we shall not be able to decide which of these possibilities is correct.

It is as a clinician striving to hide his ignorance of serology that I now venture upon some consideration of the fundamental nature of the serological tests we employ. To recapitulate what is now ancient history, Wassermann applied, as he thought, the Bordet-Gengou phenomenon to the serological diagnosis of syphilis, using the fixation of complement to detect a reaction between a specific treponemal antigen and the immune bodies 
developed in syphilitic serum. In his original technique he employed as antigen an extract of the livers of syphilitic infants in the belief that the multitude of spirochaetes therein produced a specific antigen. When it was demonstrated that normal human liver, and later almost any extract of animal tissue but especially of beef heart, were antigenic, doubts as to the specificity of the antigen, and therefore of the reaction itself, naturally arose. And when, more than 30 years later, it was demonstrated that although specific antibodies to spirochaetes did exist they were not the same as the hypothetical reagin, and that a pure substance, cardiolipin, could be separated from beef heart, to make, with suitable admixture of cholesterol and lecithin, a highly sensitive and specific antigen in serological tests for syphilis, the basis of these tests as an immunity reaction seemed to have disappeared.

The objections to serological tests for syphilis as specific antigen-antibody reactions are, however, more apparent than real. Specific synthetic antigens are not peculiar to the S.T.S.; the polysaccharide antigen of the capsules of pneumococci has been separated, analysed and synthesized ; the chemical structure of some at least of the antigens of the typhoid bacillus is approximately known, and their synthesis may not be far distant. The fact that cardiolipin is an almost universal constituent of animal tissues becomes of little importance when it is recalled that only a particular combination of cardiolipin, cholesterol, and lecithin will serve as an effective antigen.

Since 1907 continuous improvements in technique have resulted in the development of S.T.S. of both high specificity and sensitivity ; applying the prag. matic principle that what works in practice must be true, it is difficult to believe that this could have been achieved on the basis of a mistaken hypothesis, and that our S.T.S. are not a measure of a specific antigen-antibody reaction.

Some recent investigations upon the fundamental nature of the S.T.S. by Puccinelli (1951) in Milan, and by D'Alessandro and others (1950) in Palermo, go a little further towards resolving the theoretical problem presented by sero-resistance. These workers have demonstrated the presence of four different antigens in the Reiter strain of spirochaete, and have identified in human syphilitic sera the antibodies corresponding to three of them, presumably due to crossimmunity between the Reiter strain and Treponema pallidum. These antibodies can be separated by fractional absorption. The presence or absence of one or more of these antibodies depends upon the stage of the infection ; Fig. 1 shows that the antibodies present in the sera of resistant cases differ from those present in early infectious cases and untreated late cases.

\begin{tabular}{l}
$\begin{array}{l}\text { Normal Tissues } \\
\text { (Alcoholic extract) }\end{array}$ \\
\multicolumn{1}{c}{ Antigens } \\
\hline Reagins in Syphilitic Blood
\end{tabular}

Fig. 1 (after Puccinelli).-Presence of antibodies in various stages of syphilis.

Before leaving the subject of the S.T.S. themselves, some further reference is necessary to their increasing sensitivity. The more sensitive the procedure (i.e. the more minute the amounts of reagin detected) the further is the serological cure of syphilis removed, and the greater is the incidence of sero-resistance. Eagle (1937) has shown that of 100 patients who manifested sero-resistance as measured by the best tests at that date, only seventy would have done so as judged by the best tests in 1920 , and only 28 would have given a positive reaction with Wassermann's original technique (Fig. 2, overleaf). The influence of the sensitivity of the tests employed is shown even better in Figs 2(a) and $2(b)$, prepared from my own material, in which three tests of differing sensitivity have been carried out on the same samples of blood (see next page). It is thus apparent that sero-resistance is at best a relative matter.

Turning now to the interpretation to ue placed on sero-resistance in the practical management of the patient, an appeal to the authorities reveals a fair degree of unanimity. Thus, Harrison (1950) writes :

The difficult problem is to decide when to stop treatment. Many syphilologists will not treat these patients 


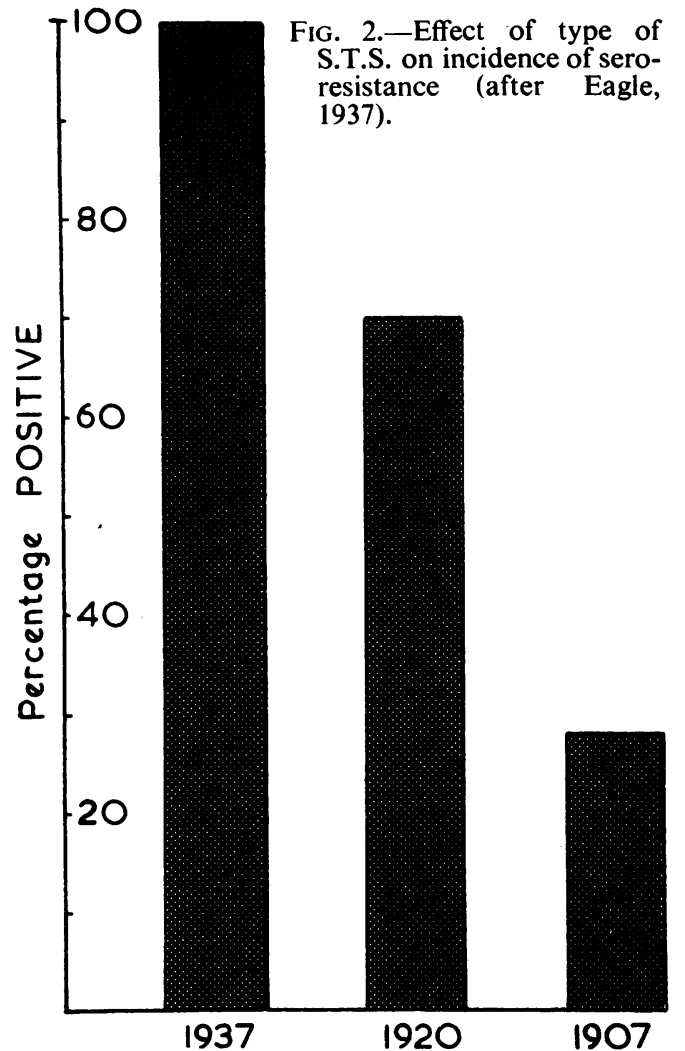

beyond the stage when they show no clinical sign, maintaining that the treatment only gets on the patients' nerves and so forth. I am sure that this view is mistaken. Patients with latent syphilis who have not complained of any particular feeling of ill-health commonly remark after the first or second course of treatment that they now feel better than they have done for years ; it is as if an insidious depressor of health had been removed. Moreover it is not true that the treatment of these cases has no effect on the serum reactions. If a good quantitative serum test is applied, expressing the strength by the number of units, the reduction in the number of these is usually most striking after the first two or three courses. If the patient is still relatively young, I usually treat until the strength of the reactions has shown no change for two or three courses, and then advise continuation at the rate of three courses in two years. Frankly, in any given case, treatment may not be necessary after the first few courses have been given, but nobody can say whether this is or is not so at the time the patient is under it, and the treatment is given as an insurance against insidious damage by the spirochaete.

Lloyd (1949), writing on the treatment of latent syphilis states:

The type of treatment advised depends to a great extent upon the age of the patient and upon an estimate
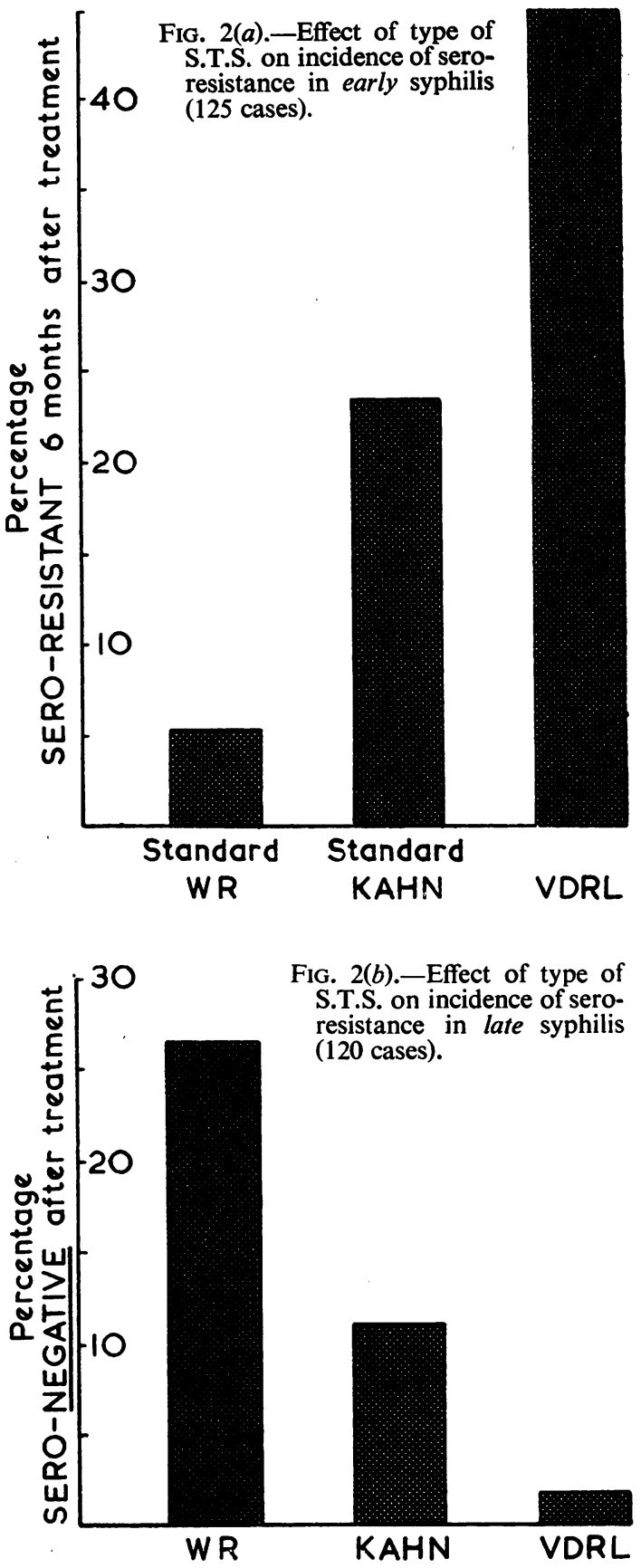

of the duration of the infection. ... The positive serological tests are usually reversed very slowly by treatment and several courses (of penicillin, arsenic, and bismuth), with intervals, may be required. In some cases the serological tests remain obstinately positive in 
spite of prolonged treatment, but it is thought that treatment over 12 to 18 months will be sufficient to perpetuate the latency and to prevent any later disasters. The attainment of a negative serological test within 3 to 6 months suggests that the infection has been present for a year or two only. ... In established tertiary syphilis the positive serological reactions will usually remain unaltered by treatment, and should not be taken as a guide to progress or further treatment ; in fact, further tests are best omitted.

Lloyd (1950) points out that in tertiary syphilis it is more important to treat the patient than the disease :

The positive serological tests in later life will remain unaffected by any form of treatment.

Batchelor (1949), writing on the treatment of late syphilis, states that :

Further treatment may be guided by the clinical response and perhaps by the serological improvement, if any. In elderly patients the object to be aimed at is always the improvement and maintenance of the patient's health at the highest level, and never the quick reversal of the S.T.S. Certainly a decline in titre of the S.T.S. is to be welcomed, provided it does not coincide with a decline in the patient's strength and well-being. A patient may be kept feeling and looking remarkably fit in spite of his S.T.S. remaining strongly positive; in fact, blood tests may be of relatively little importance in these cases, and should never be allowed to become, through constant harrowing repetition, an obsession for both patient and doctor alike.

From the same source Lees (1939), writing on the management of late syphilis, says :

The general health is regarded as the principal guide to treatment, rather than the result of the W.R. Elderly patients with a persistently positive W.R. should not be subjected to the risks of intensive or prolonged antisyphilitic treatment.

McLachlan (1945), on the treatment of late generalized syphilis, writes :

These cases in which the W.R. remains positive (after 2 years' treatment) present the difficulty that while there is serological evidence of the persistence of a focus of $T$. pallidum infection in the body, there is no clinical evidence of the disease. The persistence of a positive W.R. does not imply that the disease is active or progressive, or that it constitutes any immediate danger to life or good health.

A final quotation from British sources comes from Marshall (1944) on sero-resistance :

Reversal of positive blood-tests is generally more difficult in late than in early syphilis. In late syphilis sero-resistance is not quite so important, and indeed, after cessation of treatment, reversal may eventually occur spontaneously. A change in therapy can sometimes effect serological reversal. In sero-resistant as in other cases of syphilis a fundamental is to remember that it is the patient and not the blood-test who is to be treated.

Turning now to American sources, the most comprehensive surveys of the problem of seroresistance have been made by Moore and Padget (1938), and again by Moore (1946).

On the genesis of sero-resistance these authors consider that :

In cases of late syphilis sero-resistance may result entirely from the persistence of well-established immunity. In patients with late syphilis the situation is entirely different [from that in early syphilis]. Sero-resistance is a usual or expected eventuality with many forms of late syphilis (Fig. 3). Studies have shown that the incidence of sero-resistance is not influenced by the scheme of treatment employed, or related to the existence of involvement of the central nervous system per se. Instead it may be viewed as an integral part of the disease.

On the significance of sero-resistance, Moore and Padget demonstrate the lack of relationship between

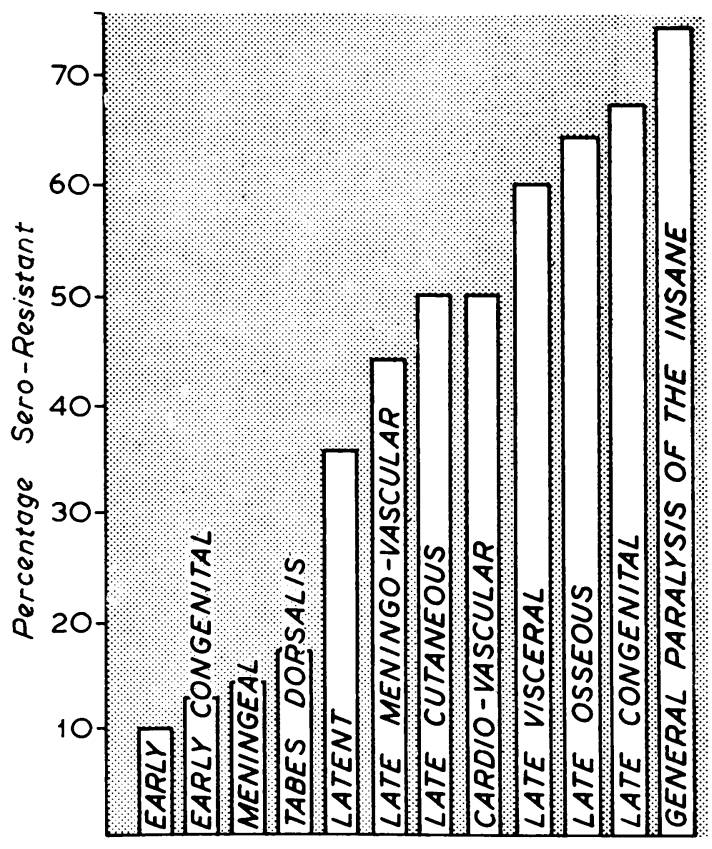

FIG. 3.-Effect of stage of syphilis at beginning of treatment on incidence of sero-resistance (after Moore and Padget, 1938). 
the incidence of progression or relapse and seroresistance (Fig. 4) :

For each type of late syphilis considered, the incidence of progression or relapse is essentially the same among patients who are sero-resistant as among those who are not. . . In patients with late syphilis sero-resistance is so common that the impossibility of placing special interpretation upon its occurrence is readily apparent.

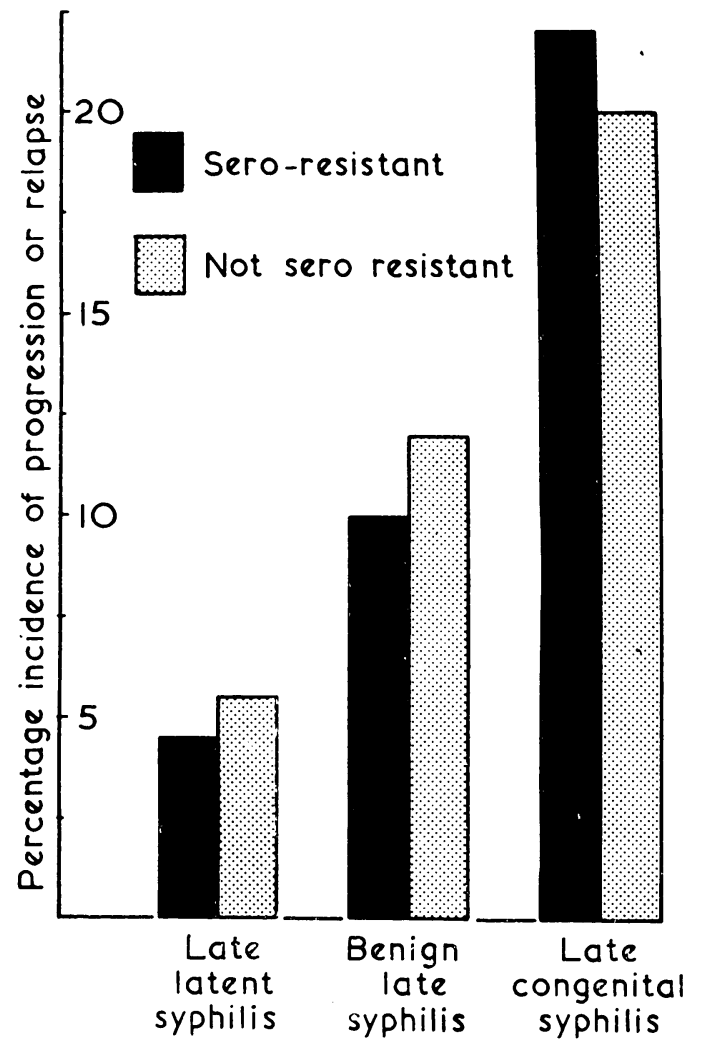

FIG. 4.-Lack of relationship between sero-resistance and the incidence of progression or relapse in patients with various forms of late syphilis (after Moore, 1946).

On the management of sero-resistant syphilis, they consider that if the healing of lesions, the relief of symptoms, the maintenance of good health, and the prevention of progression or relapse can be accomplished, success or failure in serological reversal is, or should be, a matter of complete indifference to physician and patient alike. In patients with late syphilis their ultimate clinical fate certainly cannot be measured in any degree by what happens to their blood-tests. Long term observation of large numbers of similar patients given varying kinds and amounts of treatment for varying periods of time indicates that the sero-resistant patient, adequately treated, does as well as, or even better than, his supposedly more fortunate brother with serological reversal. Considered in this light, the serological control, so far as blood-tests are concerned, of treatment in cases of late syphilis is unnecessary, even undesirable, and sero-resistance loses its fearsome significance.

Finally, and particularly to be condemned as unproved and unnecessary, and as imposing an unjustified risk on the patient, are efforts at reducing a positive reaction to negative by non-specific methods, especially artificially induced fever.

Stokes (1944), after a judicial review of the authorities, expresses the belief that the importance of the positive W.R. in old age and of the positive W.R. as such, as a manifestation of syphilis in the established infection, should be minimized. He feels that many of these elderly persons could well congratulate themselves that they had not been subjected to a treatment test of reversibility which might easily have injured their viscera and left them in a much worse state than that of possessors of mere positive serological tests. The consideration of clinical indications and good sense will allow age and time factors to decide the issue of treatment rather than the serological test as such. These views Stokes shares with Ravaut, whose work he quotes.

Thomas (1949), discussing the interpretation of serological tests, states that:

After two years of infection, patients vary greatly in the manner in which their quantitative S.T.S. respond to treatment, and they also vary in the time required for the S.T.S. to become negative. Some patients had marked decreases in reagin following rapid treatment of late syphilis and others had not. As long as the trend of reagin titre was downward and there were no pronounced sustained rises in titre from previous levels, re-treatment has not hastened the reversal of positive S.T.S. to negative. We have found malaria, artificial hyperpyrexia, arsenical drugs, and penicillin to have no appreciable effect in reversing positive S.T.S. to negative in patients who had had adequate previous therapy. . . It is important for those concerned with the management of syphilis to realize that the main purpose of treatment of late syphilis is not to obtain negative S.T.S., but to arrest whatever active syphilitic process may be present. In 70 to 80 per cent. of cases of late syphilis, no amount and kind of additional treatment will reverse positive to negative within 5 years. The proportion of patients who become sero-negative in a given time after treatment of late syphilis is of small importance.

Schulmann and Lévy $(1930,1932)$ initiated the major discussion in the French literature. They attached little clinical importance to sero-resistance, and were opposed to extended treatment, preferring observation and serological tests at 3-monthly intervals with a cerebrospinal fluid examination annually. 
In the discussion which followed their second series of papers they were supported by the majority of the speakers.

German opinion, collected by Jadassohn (1929) and in a later symposium by Hämel and other workers (1934), agrees on the relative unimportance of Wassermann-fastness, and makes it entirely a matter of individual judgment whether or not such patients shall be periodically treated, and allowed to marry, or otherwise to conduct their lives without reference to their serological status.

I have sometimes had the vague clinical impression, so often unreliable and insusceptible to proof, that antisyphilitic treatment was stimulating and prolonging a positive serum reaction, in a manner analogous to the discredited provocative test. This impression is confirmed by Stokes, who describes it as not a rare experience, and regards it as an indication for thorough and persistent treatment.

After the views which I have quoted the question of the treatment of sero-resistance should not arise. The many and varied remedies (foreign protein injections, ultraviolet light treatment, autohaemotherapy, intravenous sodium iodide, hormones, fever therapy) are themselves a measure of their ineffectiveness. Burke combined them all into a blunderbuss prescription for the treatment of reaginfastness occupying 52 weeks, at the end of which he says :

If this fails, all efforts to change the serology should be abandoned.

I do not know how many of you have had the temerity to embark upon such a hopeless project, but for cosmetic reasons two of my sero-resistant patients agreed to submit themselves to this ordeal. They are still sero-positive after its completion.

Alechinsky (1948) claimed that calciferol is capable by itself of rendering negative a refractory or irreducible sero-reaction in syphilitics who had undergone an abnormally long specific treatment. In the light of the many similarities between tuberculosis and syphilis, and of the dramatic results obtained from the use of calciferol in the treatment of lupus, this seemed a promising line to follow up. Half of a group of twenty sero-resistant patients were given calciferol by mouth, and the other half by intramuscular injection; but no changes in their serological reactions were observed.

\section{Summary}

(1) A positive serological reaction, with appropriate safeguards, means that the patient has been infected with syphilis at some time in his life.

(2) The positive serological reaction is nevertheless not a diagnosis but a stimulus to further investigation.
(3) The behaviour of the S.T.S. under treatment is not a guide to treatment, but merely an indication of the probable duration of infection.

(4) In late syphilis it is not to be expected that the S.T.S. will become negative as a result of treatment.

(5) The aim of treatment in late syphilis is not to produce a negative S.T.S.; attempts to reverse a positive S.T.S. to negative by treatment are doomed to failure because the attempt is misconceived.

\section{REFERENCES}

Alechinsky, A. (1948). Arch. belges Derm. Syph., 4, 146, 151.

Batchelor, R. C. L. (1949). In “ Text-book of Medical Treatment", 5th ed., ed. D. M. Dunlop, L. S. P. Davidson, and J. W. McNee. Livingstone, Edinburgh. Blumenthal, F., and Mallinckrodt-Haupt, A. von (1929). In J. Jadassohn, "Handbuch der Haut-und Geschlechtskrankheiten ", vol. 15, pt. 2. Springer, Berlin.

Bruusgaard, E. (1929). Arch. Derm. Syph., Wien. 157, 309.

Burke, E. T. (1942). “ Modern Treatment of Venereal Diseases". Bale, London.

D'Alessandro, G., Oddo, F. G., and Dardanoni, L. (1950). J. vener. Dis. Inform., 31, 314.

Eagle, H. (1937). Quoted by Moore and Padget (from "The Laboratory Diagnosis of Syphilis ", Mosby Co., St. Louis).

Hämel, J. ; Miescher, G. ; Mulzer, P. ; Riehl, G. ; Scholtz, W. ; Spiethoff, B. ; Stumpke, G. ; Zoon, J. J. (1934). Derm. Wschr. (symposium), 99, 892.

Harrison, L. W. (1950). In "A Text-book of the Practice of Medicine", ed. F. W. Price, 8th ed. Oxford University Press, London.

Jadassohn, J. (1929). "Handbuch der Haut- und Geschlechtkrankheiten ". Springer, Berlin.

Kolmer, J. A. (1938). Amer. J. Syph., $22,426$.

Lees, R. (1939). In " Textbook of Medical Treatment ", 1 st ed. ed. D. M. Dunlop, L. S. P. Davidson, and J. W. McNee. Livingstone, Edinburgh.

Lloyd, V. E. (1949). Practitioner, 162, 349. (1950). In "Savill's System of Clinical Medicine", 13 th ed., ed. E. C. Warner. Arnold, London.

McLachlan, A. E. W. (1945). " Handbook of Diagnosis and Treatment of Venereal Diseases", 2nd ed. Livingstone, Edinburgh.

Marshall, J. (1944). "The Venereal Diseases". Macmillan, London.

Moore, J. E. (1946). Amer. J. Syph., 30, 125.

-, and Padget, P. (1938). J. Amer. med. Ass., $110,96$.

Pesare, P. J., Bauer, T. J., and Gleeson, G. A. (1950). Ibid., 34, 201.

Puccinelli, V. A. (1951). Ibid., 35, 340.

Rosahn, P. D. (1946). J. vener. Dis. Inform., 27, 293.

Ravaut, P. Quoted by Stokes (1944).

Scholtz, W. See Hämel and others (1934).

Schulmann, E., and Lévy, G. (1930). Ann. Derm. Syph., Paris, 7 e Ser., 1, 196.

(1932). Bull. Soc. franç. Derm. Syph., 39, 961.

Stokes, J. H. (1944). In "Modern Clinical Syphilology", by J. H. Stokes, H. Beerman, and N. R. Ingraham, 3rd ed. Saunders, Philadelphia.

Thomas, E. W. (1949). "Syphilis : its Course and Management". Macmillan, New York. 